\title{
Virtual reality technology in product design and manufacturing
}

- The design and implementation of a course for the graduate study

\author{
Qingjin Peng \\ Department of Mechanical and Manufacturing Engineering, \\ University of Manitoba, Winnipeg, MB, R3T, 5V6, Canada \\ pengq@cc.umanitoba.ca
}

\begin{abstract}
This paper describes the experience of teaching a graduate course in Mechanical and Manufacturing Engineering Programs at University of Manitoba, Virtual reality technology in product design and manufacturing. The course has been delivered six years since 2001. The course provides an opportunity for students to plan and optimize a design or manufacturing process in virtual environments. Students are expected to analyze some complex, openended questions in virtual environments for conceptual design solutions. This paper introduces the course outline and teaching materials developed in the last few years. The emphasis and challenge in the teaching and learning will be discussed. Examples of course projects completed by students are presented. The further work and direction of the course improvement will also be addressed.
\end{abstract}

\section{Introduction}

Virtual reality (VR) provides a computer-generated environment for product design or manufacturing process simulation. VR integrates technologies of computers, information, image processing, communication and control with features of immersion, interaction and information intensity. VR technology has been successfully applied in a wide area including entertainment, product development, space exploration, health care and military simulation. Engineering is an important field of VR applications.

The traditional iterative product analysis leads to a long product development time and a high cost. Usually, an important product design is not finished until after a series of life-sized wooden mock-ups are built and reviewed. For example, when NASA designed the space shuttle, it had to create an entire wood and plastic life-sized model of the ship to see what it would be like. VR utilises advanced computer and visual technologies to provide a virtual environment for product development, by which product design and manufacturing can be considered simultaneously. As a result, users can minimize the use of expensive physical mock-ups and shorten the period for the product development [1].

VR has been identified as new opportunities for industry to increase the efficiency of product design and manufacturing. VR application examples include computer-aided design [2], robotics [3], assembly planning [4], manufacturing layout [5], manufacturing simulation [6], and product maintenance [7]. VR has shown its powerful for the need of large number of simulators, for interactive visual fields and for the analysis systems of both technical and human performance.

Graduate students in engineering deal with a number of design projects in their study. Design tools learnt are common CAD systems, such as AutoCAD, Pro/E, and Solid Work. These systems provide very useful tools to assist students for product drawing and structure design, but they are not good for an interactive evaluation and detail research of the design. VR provides a tool in 3D interactive environments to allow a design to be explored exhaustively. It is necessary for engineering researchers to have the VR knowledge for using VR in engineering applications.

According to a survey [8], until March 2007, there are 267 universities in the world are delivering VR courses to students. There are 78 in the US and 9 in Canada. A VR course was designed and implemented for the graduate study in the Department of Mechanical and Manufacturing Engineering at University of Manitoba in 2001. The course materials have been improved and updated in each year's teaching. The course provides an opportunity for students to plan and optimize product design or manufacturing process in virtual environments. The course introduces VR software and hardware, virtual environments, virtual prototyping in product 
development, VR data exchange and VR system developing platforms. Methodologies and tools are discussed to support the verification and validation of VR systems. VR is used as a tool to measure and compare actual performance of a design or manufacturing process. Issues related to VR and its applications in product design and manufacturing are emphasized in the teaching and learning.

\section{VR knowledge and contents for delivery}

There is a variety of definitions for VR, but they are similar, such as a computer-generated surrounding, an interactive three-dimensional (3D) computer graphical interface, or an immersive interactive environment [9]. The feature of VR can be summarised as "three I's": immersion, interactivity, and information intensity [10]. The meaning of immersion is that the VR user has a feeling to locate in a real world, but actually it is a computer generated virtual world. The interactivity comes from advanced input/output (I/O) devices that provide much powerful interactive ability than conventional CAD systems. The information intensity is that a VR system uses all information related to the human sense. Therefore, VR technology is a natural extension of 3D computer graphics with advanced input and output devices. VR users can interact with a simulated immersive 3D environment in real-time through multiple sensorial channels.

A VR system consists of three parts: VR engine, VR database and model base, and I/O devices. The VR engine is a graphic modeling and processing system. It is for object modeling, texturing, mapping, lighting, rendering and finally displaying 3D scenes in realtime. It is the infrastructure of VR. The VR database and model base stores virtual objects that are ready to be loaded in the scene whenever required. I/O devices provide the human-machine interface for the VR system control and communication. Therefore, based on the VR definition and components, VR knowledge can be delivered from three aspects: VR hardware and software, VR modelling, and VR applications.

\subsection{VR hardware and software}

VR hardware consists of VR input and output devices. VR input devices commonly include motion trackers for tracking a motion processing, and data gloves for VR system operations. There are different types of tracking sensors available including magnetic, optical, mechanical, ultra-sonic, inertial, and gyroscopic sensors. The choice of these trackers is based on their measurement volume, speed, accuracy, range, noise and distortion latency. The data gloves are used to replace keyboard and mouse in the VR operation. Its performance can be measured by its operation dimension, accuracy, and practicality.

VR requires graphic, sound and haptic or force output. The graphics display is the most important factor in a VR system. Depending on the user, there are personal displays and large Cave-type display systems available. The commonly used personal display devices are head mounted displays (HMD), others include 3D binoculars, booms, virtual windows, and auto-stereoscopic displays [11]. The large volume displays consist of one or more large projector-based screens. The formation can be Workbench, Wall-type displays, Domes or Cave-type displays. The stereo displays are formed by either active stereoscopic or passive stereoscopic projection. The performance can be measured by their resolution, colors/monochrome, field-of-view, contrast, brightness, and image distortion.

VR software is used to design, build, and maintain virtual worlds. There are two types of software systems as follows:

1). Open source toolkits such as VRML [12], Java 3D, X3D, OpenSG, and others.

2). Commercial Toolkits such as Eon Reality, Oxygen (SGI), dVise, Sense8, Delmia, and others. These tools provide different support levels based on the users capabilities. They are used based on compiled libraries, scripted language or graphical interface.

\subsection{VR modeling}

VR modelling includes geometric modeling, kinematics modeling, physical modeling, and behaviour modeling [11]. The topics of geometric modeling include: virtual world space definition; virtual observer location; perspective projection; 3D modeling and clipping; 3D space curves; 3D boundary representation; geometrical transformation; and modelling transformations for translate, scale, reflection and rotation. The topics of physical modeling include: illumination models; reflection models; shading algorithms; radiosity and realism; and collision detection. The topics of kinematics modeling include: picking, flying; scaling virtual environments (VEs); and the dynamics of numbers. The topics of behaviour modeling include: free-form deformation; shape and object inbetweening; the animation of objects; animating the virtual environment (VE); particle systems; interactive using navigation, selection, or manipulation for a convincing simulation.

\subsection{VR applications}

VR technology provides an integrated approach including feasibility analysis, iterative design, and systematic evaluation in product development. The 
applications related to product design and manufacturing include: digital rapid prototyping; design reviews; human factor studies/ergonomics studies; digital assembly; learning and training; manufacturing process simulation; tele-operation and Web-based applications.

\section{The course design}

\subsection{VR concept}

VR is an integrated multi-discipline system. A VR course should introduce following basic concepts to students.

- VR provides a high level interaction and intelligent interface.

- $\quad$ VR is good to implement tasks that may be too difficult, too complex, too dangerous, too expensive, or not yet in existence in real life.

- VR can be for something that is impractical to experience directly.

- VR can provide what is close to real life experience.

- VR can accelerate understanding and reduce uncertain.

- $\quad$ VR users can see thing that cannot be seen in a standard 3D model.

Therefore, following VR benefits are emphasized in the course delivery:

- VR allows users to discover new ways of experiencing real world and simulating complicated manufacturing processes;
- VR can integrate whole product design and manufacturing processes into a virtual environment;

- VR can reduce the product lead time, time to market and cost;

- $\quad$ VR can improve product quality and reliability.

\subsection{The course outline}

A course homepage is developed for the course teaching and learning as follows:

http://www.umanitoba.ca/faculties/engineering/mech and ind/prof/peng/teachvr.html. The course notes and tutorial materials are available on this Web page. They are dynamically updated during the course term. There are thirteen weeks for the course study. A three-hour class is scheduled per week for the course delivery. The course consist two parts, the lecture and VR systems demonstration. The details are shown in Table 1.

The grading components consist of three parts: VR software Eon Reality practice and presentation (30\%), literature review report (30\%), and VR project (40\%). The practice and presentation of VR software Eon Reality takes five weeks. The students are required to learn the VR tool, Eon Reality, to create a small VR model and to operate the model in the Eon Reality system. The writing report is based on literature review of the interesting topic. The topic is designed to extend the VR knowledge by reading and research for the understanding of fundamental theories and techniques of VR. The solutions of the report are used a guideline for the VR project that is designed based on student's thesis topics. Following Section lists the report topics used in the last two years.

Table 1. The course schedule

\begin{tabular}{|l|l|l|}
\hline \multicolumn{1}{|c|}{ Date } & \multicolumn{1}{c|}{ Lecture } & \multicolumn{1}{c|}{ Demonstration } \\
\hline Week 1 & The course outline. VR lab introduction & Eon Reality system VR models \\
\hline Week 2 & Introduction to VR and its applications & Eon Reality system: Quick start and examples \\
\hline Week 3 & Virtual terminologies, VE and VR engine & Ron Reality system: tutorials \\
\hline Week 4 & 3D computer graphics (1) & VR applications videos \\
\hline Week 5 & 3D computer graphics (2) & Data exchange. Data glove and tracking system. \\
\hline Week 6 & VR geometric modeling & Eon script programming and examples \\
\hline Week 7 & VR geometric transformation (1) & Stereo systems setup; DELMIA VR systems. \\
\hline Week 8 & VR geometric transformation (2) & VR project 1 presentation \\
\hline Week 9 & Animating the VE & Delmia digital manufacturing system \\
\hline Week 10 & VR open sources & Virtual reality modeling language (VRML) (1) \\
\hline Week 11 & Introduction to distributed VR & Virtual reality modeling language (VRML) (2) \\
\hline Week 12 & Reverse engineering for VE creations & 3D scanner and CMM operations \\
\hline Week 13 & $\begin{array}{l}\text { Image file formats in VEs } \\
\text { Level of details }\end{array}$ & $\begin{array}{l}\text { Geomegic Studio/Qualify systems for 3D shape } \\
\text { construction and comparison. }\end{array}$ \\
\hline
\end{tabular}




\subsection{Writing report topics used in the last two years} 2006

- $\quad$ Product maintenance using VR;

- Fixture/Jig analysis using VR;

- Human-centered manufacturing system with VR;

- Integration of product design and product planning using VE;

- $\quad$ Product assembly/disassembly using VR;

- VR in product quality control;

- VR in AGV simulation.

2005

- The research and development of VR open sources;

- The performance evaluation of VR open sources (VE-suite/OpenSG/VR/Juggler/FreeVR/Open Inventor) comparing with Eon Reality;

- The development of VR geometric modeling techniques;

- The networked techniques to support distributed VR systems;

- VR physical modeling techniques and methods;

- VR behavior modeling techniques and methods;

- VR model management techniques and methods;

- VR manipulation/animation techniques;

- VR visualization technology;

- Data transfer problems and solutions between CAD and VR systems.

\subsection{Report guideline}

- Each student selects one of the topics (not repeatable), and discusses it with the instructor;

- $\quad$ Each student is required to submit a written report with the printed format;

- Each student has to investigate at least 10 research papers from journals and conference proceedings published in last two years to cover the latest development of the topic;

- The report is a summary of all papers reviewed. Don't copy the sentence from the literature, do organize and summarize the description from readings. Any comment and data from the literature must be clearly addressed;

- Following aspects should be emphasized in the report:

1) What are the special needs for VR in the filed?

2) What is the related research in this area?

3) Analyze the research from the methods used, functions and solutions provided, questions remained and development trend.

\subsection{VR project}

The project is designed for students to develop their skill in VR design and applications. There are following guidelines and requirements.
- Develop a VR application on the chosen topic based on the individual research interest;

- VR tools used can be Eon Reality, Delmia VR systems, or other tools available;

- The project should take advantages of VR, such as $3 \mathrm{D}$ visualization and interaction;

- A user-friendly interface should be developed in the project. This may be done by using interactive menu and buttons.

- Wrap the project into an HTML file linking to:

o the VR model

o the Readme file (about 1000 words)

- Hand in the program code in a disk or CD;

- Students demonstrate the project before handing in the final project documents.

Note that the project must be the original work. If the project contains externally generated code, or components of a complex geometrical model imported from others' work, it should be mentioned clearly in the report so that the personal effort can be clearly justified.

The VR project is marked by a peer evaluation with the following criteria:

- If the definition and objective of the project is clear;

- If it is a complete project;

- If the project has significant and useful output;

- If the presentation is clear;

- If the question answer is correct.

Table 2 shows selected the VR projects done by students in the last few years.

\section{The lab support}

The course is delivered in the VR lab. This enables the instructor to deliver the course materials and to demo the VR devices for students in the class. The lab used for the course teaching and learning is the Virtual Manufacturing Centre (VMC) in the Department of Mechanical and Manufacturing Engineering at University of Manitoba. The VMC was established in 2002 by the support of Canadian Foundation for Innovation (CFI) Grant to enhance the research and innovation in product design and manufacturing using VR technology. The lab has equipped EON Reality and Delmia digital manufacturing systems, which use a $2.4 \times 2.4$ m back-projected stereo wall for the 3D graphic display. The 3D stereo display system can produce a good imagery and can present well for a group of VR users. The system integrates a Head Mounted Display with Data Gloves and motion tracking. Users can be completely immersed into the environment interacting with the gloves while tracking head and hands' motions in six degrees of freedom on 
the $2.4 \times 2.4 \mathrm{~m}$ screen. The lab also includes a 3D laser scanner and VR development workstations with software that can import 3D formats from other CAD systems. They can help to quickly create a new VR environment.

The lab has provided a strong support for the VR course teaching and leaning. For the need of VR facilities, different applications may emphasize different functions. Manufacturing modeling, behaviour, and collision detection are important features for a virtual manufacturing system. In order make up the lack of the Eon Reality system, Delmia VR system is chosen as an additional software tool to meet the need in the course project. Delmia system is a suite of digital manufacturing software. The software focuses on the need of manufacturing with solutions to plan, define, create, validate and control manufacturing systems. Following software packages are selected for the need of the course project.

Table 2. Selected examples of the VR projects

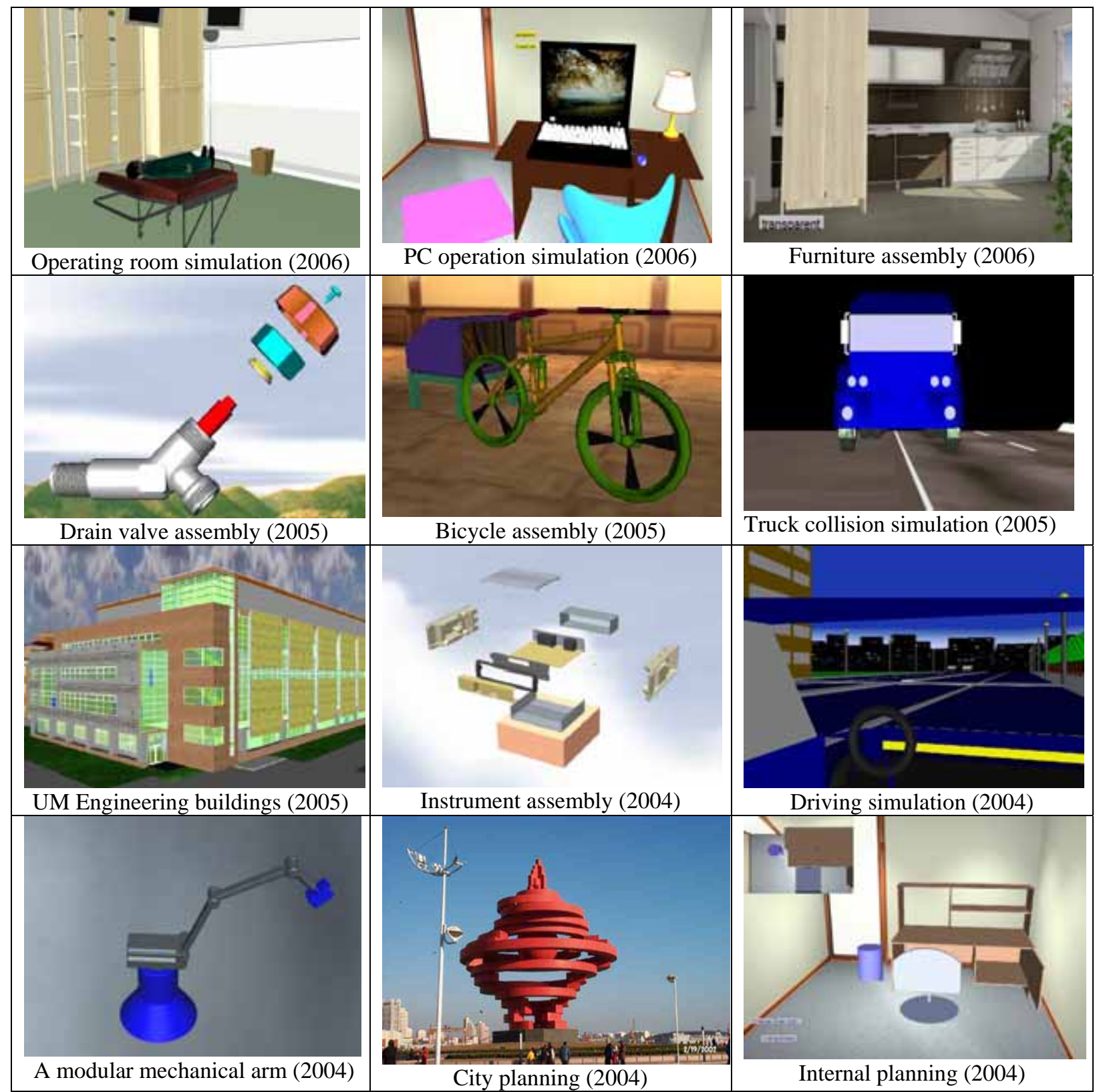


IGRIP is a robotic simulation tool for robot design, evaluation, and off-line programming. It can incorporate with real-world robotic and peripheral equipment, motion attributes, kinematics, dynamics and I/O logic. It can optimize robot locations, motions and cycle times, and detect collisions between robots, parts, tools, fixtures and surroundings.

Virtual NC is a simulation tool for machine tools, controllers and material removal. It can validate processes, tooling setups and NC production programs with real-time and 3D solids modification.

Quest is a discrete event simulation tool used to model, experiment and analyze facility layout and process flow. It provides real-time interaction, modification of model variables and viewing parameters during running.

Process Planning is a process and resource planning support environment. The resulting process diagrams can provide an overview of the sequences and links between processes and resources early in product design conception. The planning tasks include layout planning, time measurement, process and resource planning, product evaluation, and cost analysis.

DELMIA Assembly provides a geometry-based solution with the ability to link and view product data from CAD systems. It can be used to view the assembly sequences and processes, and link each process step to the manufacturing resources utilizing a user interface. The assembly process and links to the product and resource data are stored in a shared database of product, process and resource information.

\section{Problems and challenges}

There are still not many Colleges and Universities teaching VR today. It has to be justified from the technical need, barriers, cost, instructor availability and market for graduates. Some current barriers for the VR education and applications include as follows.

- Cost: VR systems, especially for immersive systems, require a significant investment in hardware. It is not affordable to most Colleges and Universities, especially just for the teaching purpose.

- User interface: There are obviously differences in the performance of VR toolkits between their demonstration and the practical applicability because of the poor capability of integration with other CAD, CAM systems. It takes a long period to construct a user-based application because the inefficient interface for data input and limited modelling method.

- Standardization and simplification: The interface of VR device input/output is a major problem because the users need special interfaces for different devices to integrate these devices into a system. The link and transform of data within these devices hamper the effect of the immersion and real-time.

- $\quad 3 D$ environment modeling process: The most VR systems use CAD toolkits as tools to generate their 3D models, such as Pro/Engineer and AutoCAD, but it takes a long time to build a new virtual environment for a special application.

- Precision: It is a conflictive in the demand of speed and precision for VEs. The details and resolutions of a VE have to be reduced in order to increase the speed of image rendering, but it decreases the accuracy of a process simulation.

- There are not good supporting textbooks and laboratory manuals for the VR teaching. VR technologies are developed very rapidly. The course materials have to be updated annually. The limited lab resources limit the number of students to take the course and do projects.

- VR deals with multi-disciplinary subjects. It requires the knowledge of computers, information, image processing, communication, automatic control and engineering.

- Engineering students do not have enough programming skills. The course has to be an application-oriented study.

\section{The special designed topics for engineering students}

Based on the background of engineering students and the need of engineering applications, following two topics are specially designed for the VR course.

\section{1). Data exchange, user interface and data communication in VR-based product design and manufacturing systems.}

Currently, VR hardware and software are developed separately without a universal standard. When selecting a VR system, not only VR hardware such as I/O devices, but also the VR software, i.e. development tools, have to be considered. VR software serves as a toolkit that provides a development environment for VR hardware to support VR applications. The VR software may be run on different operation systems, and may support different VR hardware. The architectures of VR software range from platform independent languages implemented on low cost desktop workstation to high end systems customized for the high cost computation required for immersive VEs. There is no modeling function 
available in most of VR software toolkits. An interface is needed to link modeling tools, such as CAD systems. The common CAD formats include DXF, IGES, 3DS, VRML and STL depending on CAD systems used. It is not always compatible even that some toolkits provide the import/export of these formats. The feature, color, or location of a model may be lost when formatted data are imported into VR software to create VEs. We have tested a few models using different export and import formats into VR systems, such as from AutoCAD (DXF) to Eon, from 3D max (3DS) to Eon, from 3D max (VRML) to Eon and from Pro/E (STL) to Eon. It is hard to say which one can generate a prefect result. Some repairs may need after the model is transferred to VEs, but the repair may not be always possible.

Therefore, the user interface is one of the most important issues in the VR application. The standard data exchange formats of CAD and VR systems have to be introduced in the lecture and to be practised in the course project for the students. In order to understand the need of data and information exchange in a VR system, a VE is used to introduce the data required in a manufacturing system. The VE is used as an interface between the system and users for decision-making. Data exchange between the manufacturing system and users is required during product review and production evaluation. The architecture of the system is shown in Figure 1. Based on the system presented in Figure 1, users can retrieve data associated with a manufacturing system, including the product modelling, machine layout, manufacturing processing, assembly simulation and other processing in a manufacturing system. As shown in Figure 1, data exchange and communication in a manufacturing system are required for product review, VE model generation, product model storage, and connection with Intranet network.

The product review communicates users' request to product database. Product model base provides the VE model generation with information required to construct the product model for review. It also interacts with the VE to ensure that the geometric model representation is consistent with the product definition. A two-way data exchange between the product review and product database is required to communicate changes. The adaptive nature of the product review requires the data exchange with the product review to obtain information and to determine modifications of the product model. Since the VE model generation needs detailed geometric information, this information is obtained from CAD data. The product review is responsible for providing information for the construction of VE. Details of Figure 1 are described as follows.

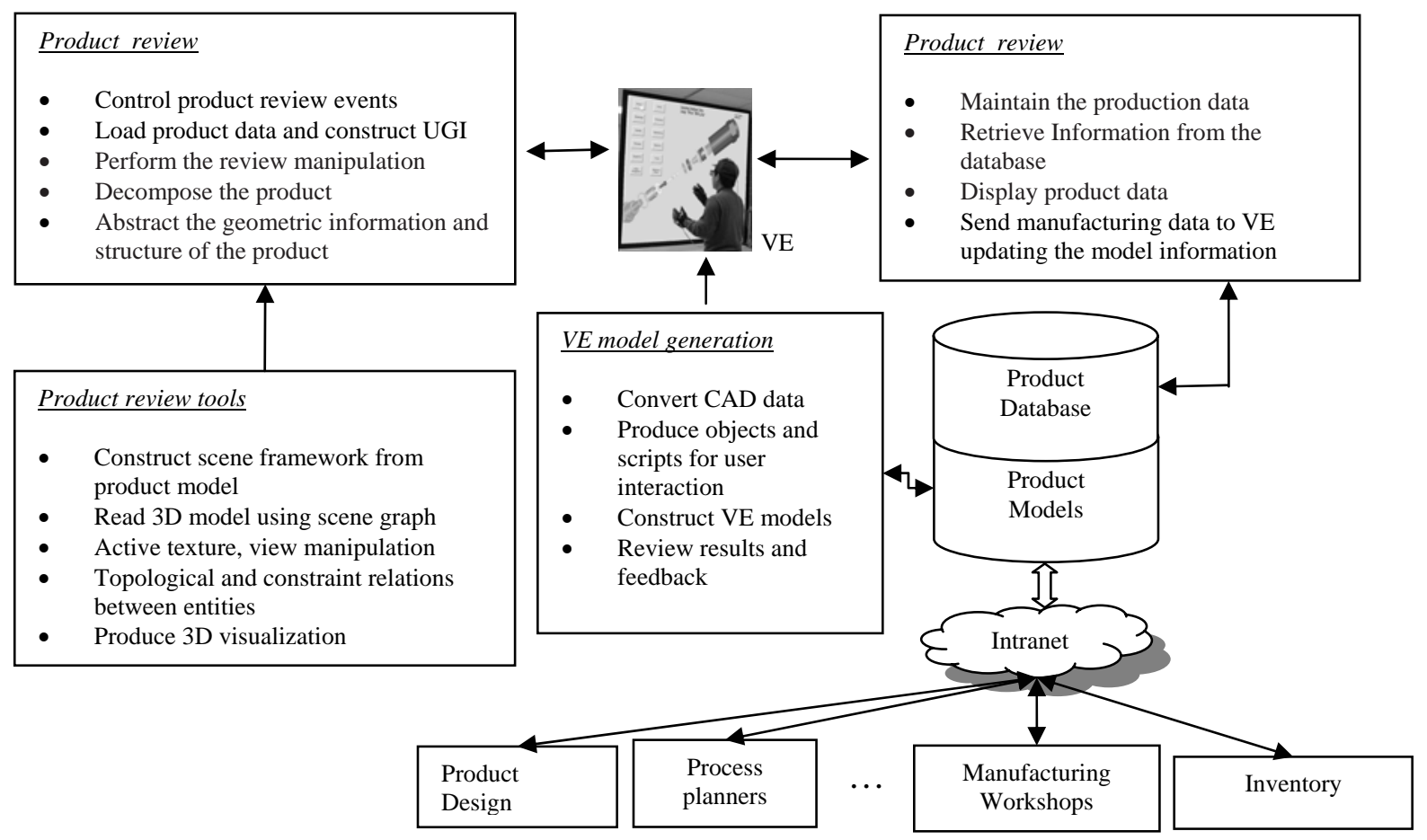

Figure 1. Data exchange in VE-based manufacturing applications 
Product review provides the event control for the product 3D model review to perform the review manipulation. It abstracts the geometric information and the structure of products, and decomposes the product into the level of details for information to support the operation of product evaluation and verification. The model review manages products at the assembly level. When a product is chosen to review, the model review will first retrieve the assembly model of individual components or subassemblies that are defined in product design. The VE starts from the product assembly model with an associated operation menu. To support a high-level definition of the product for the review processing, the component model is a hierarchal description of the product. The component includes a set of attributes and the propagation of attributes associated with it governed by a set of rules. It also maintains production data and information for the functionality required to perform the product evaluation. The product bill of materials follows the tree structure of product data. Production information and data are stored in the table form. Microsoft Access, a relational database management system, is used for the introduction of the relational database management. The database consists of data tables. The data in each table can be queried through relations. The data tables are inter-related through the one to many or many to one relationships defined by the product.

The product review uses the analogy to query data in databases to address the access to field information. Information retrieved from the database includes the product bill of materials, process plan, storage statement, supply chain and delivery details and others associated with the interesting product evaluated.

Product review tools are responsible for generation of the appropriate product model, for selection of display technologies, for provision of VE creation tools, for evaluation of the models obtained, and for the decision of improving the model. The first step for a product review is to determine techniques used to perform the review processing. At a minimum, this includes ascertaining the VE function and technique that can be applied to display the product model. The product model then is examined if it is complete enough to apply one or more of possible sets of modeling and evaluation methods. The review control is through either the geometric or component model of a spatial relation. The relation is a key to the effective operation of the product model review. Eon Reality is used as a tool for the demonstration. The link between the product model and production data can be created by VB script in Eon Reality in the course project.

$V E$ model generation constructs product models for the VE to define the state of the model and information provided by a product design. The integrated system of CAD and VE today is typically limited to a one-way transfer from CAD to VR. An interaction based on the VE is introduced for the communication of the review result to the design in the coupling of the analysis procedure.

The virtual environment (VE) is an interface for users to communicate with products. The interactive manipulation can use tracking sensors and data gloves associated with display devices to generate a product review and operation simulation. The VE is introduced using Eon Reality system. The VE can be generated by product review tools, which can produce static displayed objects and scripts for users' interaction. The static product models can use any standard data exchange formats which are imported into Eon Reality system to create VEs.

Data collection and update is supported by an Intranet network to distribute data in an organisation. Considering the data distribution in a business, product design and production information can be accessed through an Intranet network. The VE for product data review can be located in a VE centre, data in the database may be located at different departments and workshops for the data use and update. It allows the distribution of product data within a business through the Intranet. When users view and operate a design model on the VE, the related data or models are retrieved. The system searches for related data, models and information, returns searched results to the VE scene, and displays them.

There are some plug-in toolkits available for free download from Internet to support a 3D navigation on Web browsers. One of these toolkits can simply installed as a plug-in interfaces in the computer to review VR models, such as Cosmo plug-in for VRML model and Eon plug-in for Eon Reality models. These plug-in toolkits provide a simply way to view 3D models without relying on expensive VR I/O devices. It is also a useful tool to develop Web-based 3D simulation systems [13]. The model retrieval-based modeling is an efficient way to share the model in different users through a network system. In order to share the model and data to develop a networked VR application, the function to support the networked VR applications is also introduced in the class.

\section{2). Reverse Engineering technology for VE construction.}

The first important work of VR applications is to create virtual objects for VEs. There are different modeling methods to create a virtual world. Using CAD models to construct VEs is a common method. 
The reverse engineering method is a fast approach to obtain the geometrical information from an existing object for VEs. Reverse Engineering (RE) provides an effective tool for the VE construction. RE is widely used in various areas of product development. The recovery of the $3 \mathrm{D}$ shape of an object is the basis of RE. Coordinate measuring machines (CMMs) are traditional RE tools used in manufacturing industry. CMMs provide a high measuring accuracy and stability, but disadvantages are the contact measurement and a point-to-point slow measuring process. There are a number of factors that affect the digitizing uncertainty of CMMs, such as travel speeds of the probe, pitch values, probe angles, probe sizes, and part sizes.

The development of 3D digital technology in recent years has improved 3D shape recovery in RE. Besides CMMs, vision-based technology has significant applications in 3D shape recovery. The technology to process $3 \mathrm{D}$ data of objects has also been remarkable improved in recent years. Advanced software and increasingly powerful computers allow a large database and fast data post-processing. 3D scanners play an important role in vision-based 3D scanning technology. There are many commercial products available in the market. However, there is not a simple solution to select and use 3D scanning techniques because of the variable performance and high expense. Based on the need of engineering applications, the VR course discusses and evaluates current 3D scanning technology, and also suggests a cost-effective 3D digitizer for VEs.

3D digitizing technology is introduced in the class based on the contact and non-contact techniques of 3D data acquisition. The contact means that the measuring probe touches the recovery surface of objects during the data acquisition. The contact acquisition obtains data using a contact measuring process. The devices include joined arms and CMMs.

Destructive and non-destructive methods are used in contact measuring process to obtain the surface which can be touched during the measurement of objects. The destructive technique gets the touched surface by cutting or slicing object into pieces. Non-destructive methods can only measure the profile of an object.

Non-contact data acquisition technology uses an energy source, such as laser, white light, microwave, radar, and ultrasonic sound, to obtain 3D data of an object without touching the surface of objects in the measurement. There are two techniques used to receive signals of the energy source from measured surface: reflective and transmissive methods. Reflective method uses a receiver, such as a camera, to get the reflecting source from the surface of objects. According to the source used, reflective devices are divided into optical devices and non-optical devices which use microwave, radar sensor or ultra sound. Non-optical systems are based on a time-delay measurement to calculate the distance traveled by sound or microwaves. Transmissive methods get a series of slices when the source is emitted through the part. These slices can be used to produce volumetric data or feature extraction. The lecture focuses on optical scanners

A general process of optical scanner-based 3D shape reconstruction is introduced to the students. Point clouds are data acquired from objects scanned by a 3D optical scanner. A few scans may be needed to acquire different views of an object. The post-processing is the most important work to generate final structure of the object after scanning. The software makes triangles using data of point clouds. The data from each scan are constructed as triangle formations, and are merged to form a polygon mesh after deleting redundant data and filling holes of the surface recovered. Then, the software generates the feature curve and NURBS curve from polygon data. Finally, NURBS surfaces form a completed 3D shape of the object after rendering. The data can be saved as a standard data format for further applications in VR systems.

There are different optical 3D scanners available in the market based on technology used. The category of optical 3D scanners can be divided into the laserbased, white-light-based and radar-based scanners. Four well-known scanners in the market are analyzed based on the performance and cost. Besides the measuring accuracy of the scanners, other performances, such as the scanning speed, easy use, system reliability, and standard of data output format, are also evaluated. ShapeGrabber scanner is available in the lab. It uses different-sized scanning heads for different measurement ranges to achieve the required volume and accuracy.

Two objects are used to show the evaluation the performance of the 3D laser scanner in order to understand the detail of its applications. The scanner's operation uses central software to control scanning process. The software can automatically detect the scanner head used on the apparatus and prompt users to use a certain calibration plate. The calibration is required when the head changes, or after scanner is adjusted, or the scanner has not been used for a long time. A configurator is used to set the scanning range, resolution, intensity of laser beam, and depth of measuring field. A completed object is usually required a few scans to obtain its whole surface. A satellite dish and a wind generator blade are selected as test samples.

The software for data acquisition and processing is an important part of 3D digitization. The experience is 
required to arrange scanning process and number of scans to get an entire surface of the object. An effective operation is discussed in the class to reduce the data size, shorten the processing time and form a complete object shape from the least scanning times.

Geomegic studio is introduced for the data postprocessing in the class. Geomagic Studio is a software tool developed by Raindrop Geomagic, Inc. It has functions including the scan registration of multiple point clouds, point cloud noise reduction, filling holes of scanned data, polygon creation, data repair and editing, and NURBS surface creation.

\section{Conclusions and further work}

Virtual reality has become an important and useful tool in science and engineering. VR applications cover a wide range of industrial areas from product design to analysis, from product prototyping to manufacturing. The design and manufacturing of a product can be viewed, evaluated and improved in a virtual environment before its prototype is made, which is an enormous cost saving.

This paper introduced the experience of teaching a VR course for engineering graduate students. The course materials and lab projects are presented. The value of the course has been shown in the student late thesis work. We will continue develop the course contents and update the course materials based on the VR technology development and the feedback from the students. Hopefully, the course can meet more requirements from undergraduate students and students from other faculties in the future.

\section{References}

[1] Q. Peng and C. Chung, “A visualised CAPP System for Agile Manufacturing, International”, International Journal of Manufacturing Technology and Management, 2007, 11(3/4), pp. 338-254.
[2] C. Cera, W. Regli, I. Braude, Y. Shapirstein and C. Foster, "A Collaborative 3D Environment for Authoring Design Semantics”, IEEE Computer Graphics and Applications, 2002, Vol. 5/6, pp. 43-55.

[3] G. Ahrens and G. Pageau, "Trends in the robotic simulation industry”, Assembly Automation, 2002, 22(3), pp. 230-234.

[4] C. Chung and Q. Peng, "A novel approach to the geometric feasibility analysis for fast assembly tool reasoning”, International Journal of Advanced Manufacturing Technology, 2006, Vol. 31, pp. 125134.

[5] R. Yaman, "Establishment and use of virtual layouts for manufacturing”, Integrated Manufacturing Systems”, 2001, 12(6), pp. 400- 408.

[6] Q. Peng and C. Chung, "A visualised CAPP System for Agile Manufacturing”, International Journal of Manufacturing Technology and Management, 2007, 11(3/4), pp. 338-254.

[7] C. Chung and Q. Peng, "A hybrid approach to selective-disassembly sequence planning for demanufacturing and its implementation on the Internet”, International Journal of Advanced Manufacturing Technology. 2006, Vol. 30, pp. 521-529.

[8] http://www.caip.rutgers.edu/vrtechnology/resources/pu blic/survey.html

[9] K. M. Stanney, Handbook of Virtual Environments, Design, Implementation, and Applications, LEA, 2002.

[10] J. Vince,Virtual Reality System, Addison-Wesley Publishing Company, 1995.

[11] G. Burdea and P. Coiffet, Virtual Reality Technology, Second Edition, Wiley, New Jersey, 2003.

[12] A. L. Amed, D. R. Nadeau and J. L. Moreland, VRML 2.0 Sourcebook, 2nd Edition, John Wiley \& Sons, Inc., 1997.

[13] S. Diehl, Distributed Virtual Worlds --Foundations and Implementation Techniques Using VRML, Java, and CORBA, Springer, 2001. 